\title{
Von der Umkehr des Matthäus-Effekts - Stundenintensive Weiterbildung bei geringen numeralen Kompetenzen
}

\author{
Luise Krejcik • Anke Grotlüschen
}

Eingegangen: 12. Dezember 2019 / Angenommen: 5. März 2020 / Online publiziert: 27. März 2020

(C) Der/die Autor(en) 2020

Zusammenfassung Das Matthäus-Prinzip wurde für die Weiterbildungsbeteiligung nachgewiesen, nicht aber für das Weiterbildungsvolumen im Kontext von Numeralität. Aufgrund der Verwobenheit mit sozialen Kontexten ist Numeralität unabhängig vom Bildungsabschluss ein relevanter Prädikator für das Weiterbildungsverhalten. Mittels eines internationalen Vergleichs, basierend auf PIAAC-Daten, verweist der Beitrag auf eine Umkehr des Matthäus-Effekts für Weiterbildungsvolumina. Im OECD-Durchschnitt und in der Mehrzahl der nordischen Länder nehmen weiterbildungsaktive Erwachsene mit geringer Numeralität mehr Weiterbildungsstunden wahr als jene mit hohen numeralen Kompetenzen. Die Ergebnisse deuten auf die Relevanz wohlfahrtsstaatlicher Kontexte für die (Um)Verteilung von Weiterbildungszeit.

Schlüsselwörter Numerale Kompetenzen · Matthäus-Effekt · Numeralität · Weiterbildungsvolumen $\cdot$ Weiterbildungsbeteiligung $\cdot$ PIAAC $\cdot$ International vergleichende Erwachsenenbildungsforschung

L. Krejcik $(\bowtie) \cdot$ Prof. Dr. A. Grotlüschen

Fachbereich Berufliche Bildung \& Lebenslanges Lernen, Universität Hamburg, Hamburg,

Deutschland

E-Mail: luise.krejcik@uni-hamburg.de

Prof. Dr. A. Grotlüschen

E-Mail: anke.grotlueschen@uni-hamburg.de 


\title{
Reversing the Matthew Principle - Adult Education in the context of low numeracy
}

\begin{abstract}
The Matthew Principle was demonstrated for participation in further education, but not for the volume of further education in the context of numeracy. Due to its interdependence with social contexts, numeracy is a relevant predictor of further training behaviour irrespective of educational attainment. By using an international comparison based on PIAAC data, the article points to a reversal of the Matthew effect for further education volumes. In the OECD average and in the majority of the Nordic countries, adults with low numeral competence who are active in further education perceive more hours of further education than those with high numeral competence. The results point to the relevance of welfare state contexts for the (re)distribution of further education time.
\end{abstract}

Keywords Numeral competences $\cdot$ Matthew effect $\cdot$ Numeracy $\cdot$ Volume of further education - Further training participation - PIAAC - International comparative adult education research

\section{Einleitung}

Mit der Metapher des Matthäus-Effekts „Wer hat, dem wird gegeben“ wird in der Weiterbildungsforschung der enge Zusammenhang von (niedrigem) Bildungsabschluss und (geringem) Weiterbildungsverhalten beschrieben. Die an die soziale Herkunft geknüpften kumulativen Bildungseffekte und damit einhergehende soziale Strukturiertheit von Weiterbildungsbeteiligung wurde empirisch überdeutlich belegt (Eckert 2010, S. 270). Für den deutschen Kontext sind insbesondere die Analysen des Berichtssystem Weiterbildung (1979 bis 2007) und dessen internationaler Nachfolgestudie Adult Education Survey (AES) zu nennen (Bilger et al. 2017). Dabei zeigen sich über die Zeit weitestgehend stabile Beziehungen zwischen formaler Bildung und Weiterbildungsbeteiligung. Grotlüschen et al. (2016) konnten auch einen Zusammenhang von Grundkompetenzen (Literalität und Numeralität) und der Weiterbildungsbeteiligung von Erwachsenen aufzeigen.

Dabei wird das Weiterbildungsvolumen - die Gesamtdauer der besuchten Weiterbildungen in Stunden - bislang wenig berücksichtigt. Da eine hohe Partizipationsrate keinesfalls zwingend mit einem hohen Weiterbildungsvolumen je Teilnehmenden einhergeht (Desjardins et al. 2006, S. 36ff.), besteht hier Forschungsbedarf. Eine differenzierte Betrachtung des Weiterbildungsvolumens ist auch deshalb relevant, weil die Ausübungsdauer einer Weiterbildung den daraus resultierenden Bildungsund Kompetenzgewinn wesentlich beeinflussen kann. Stephen Reders längsschnittliche Befunde von US-amerikanischen Erwachsenen zeigen, dass Weiterbildungen kurzfristig literale Praktiken beeinflussen, dagegen ist ihr Einfluss auf die Kompetenzentwicklung ein eher langfristiger Prozess (Reder 2005). Zudem sind ausreichende und geplante Zeitfenster wichtige subjektive Voraussetzungen für Lernende, um sich auf Lerninhalte einlassen zu können (Schmidt-Lauff 2018, S. 332). 
Im Hinblick auf Numeralität fehlt es jedoch an Diskussionen und Befunden, welche über die Betrachtung der Weiterbildungsquote hinausgehen. Der vorliegende Beitrag versucht, diese Lücke zu schließen. Er untersucht, ob der in der Weiterbildungsforschung häufig rezipierte Matthäus-Effekt im Kontext von Numeralität unter Berücksichtigung des Weiterbildungsvolumens zutrifft. Es wird insbesondere der Frage nachgegangen, inwiefern Erwachsene mit numeralen Fähigkeiten auf einem niedrigen Kompetenzniveau weniger Zeit in Weiterbildungen verbringen als Erwachsene mit hohen numeralen Kompetenzen. Ferner wird gefragt, ob Numeralität ein relevanter Indikator für die Weiterbildungschancen von Erwachsenen ist. In der Untersuchung werden non-formale Weiterbildungen berücksichtigt - organisierte Bildungsaktivitäten, die innerhalb und außerhalb des Erwerbsarbeitskontextes stehen und von Institutionen außerhalb des formalen Bildungssystems angeboten werden (Rau 2016, S. 99f.). Die Forschungsfrage wird deskriptiv und mittels Schätzanalysen überprüft. Der internationale Vergleich auf Basis von PIAAC ermöglicht eine länderübergreifende Analyse des Zusammenhangs von Numeralität und Weiterbildungsvolumen. Numeralitätsniveaus und Wohlfahrtssysteme variieren zwischen den untersuchten OECD-Ländern, ein internationaler Vergleich kann Aufschluss über die Rolle nationaler Kontexte für die Verteilung von Weiterbildungsvolumina geben.

\section{Forschungsstand}

\subsection{Relevanz und Begriffsverständnis von Numeralität}

Die Kompetenzforschung hat den Einfluss von Numeralität auf die Teilhabechancen in vielen Lebensbereichen längst erkannt (Rammstedt 2013, S. 127 ff.). Numeralität ist für die Partizipation am Arbeitsmarkt bedeutsam und spielt insbesondere für kaufmännische, gewerbliche, technische oder mathematisch-naturwissenschaftliche Berufsfelder eine große Rolle (ebd., S. $144 \mathrm{ff}$.). Ferner erhöht geringe Numeralität das Risiko von Arbeitslosigkeit und geringem Einkommen (Banks et al. 2010; Disney und Gathergood 2013, S. 19ff.); sie verstärkt die Gefahr der Verschuldung und kostenintensiverer Kreditaufnahmen auch nach Berücksichtigung soziodemografischer Merkmale (French und McKillop 2015, S. 6; Lusardi und Scheresberg 2013).

Die Relevanz von Numeralität wird in Deutschland spätestens seit der Ausrufung der „Nationalen Dekade für Alphabetisierung und Grundbildung“ im Jahr 2015 vom Bundesministerium für Bildung und Forschung und der Kultusministerkonferenz anerkannt. Im Jahr 2016 formulierten die Vereinten Nationen in der Agenda für nachhaltige Entwicklung bis 2030 (Sustainable Development Goals) die Erhöhung von Numeralität in der Weltbevölkerung als explizites Ziel: „ensure that all youth and a substantial proportion of adults [...] achieve literacy and numeracy (UNESCO 2016, S. 8).“ Dennoch sind numerale Kompetenzen und ihre Auswirkungen auf die Teilhabe am Lebenslangen Lernen bislang wenig beforscht.

Das Begriffsverständnis von „Numeralität“ (englisch Numeracy) variiert im internationalen Kontext sowie zwischen den Fachrichtungen. Die Forschungsstränge New Literacy Studies, Ethnomathematics und Situative Learning Approaches be- 
tonen die Bedeutung von Numeralität als sozialer Praxis (Yasukawa 2018). Dabei werden die soziokulturellen, historischen und politischen Kontexte, in denen numerale Praktiken stattfinden, in den Fokus genommen (ebd.).

In der vorliegenden quantitativen Analyse wird hingegen auf das kompetenzbezogene Numeralitätsverständnis von PIAAC (Programme for the International Assessment of Adult Competence) der Organisation für Wirtschaftliche Zusammenarbeit und Entwicklung (OECD) zurückgegriffen und Numeralität definiert als ,die Fähigkeit, sich mathematische Informationen und Ideen zugänglich zu machen, diese anzuwenden, zu interpretieren und zu kommunizieren, um so mit mathematischen Anforderungen in unterschiedlichen Alltagssituationen Erwachsener umzugehen“ (Zabal et al. 2013, S. 47). Numeralität umfasst folglich u. a. den Umgang mit mathematischen und quantitativen Prozessen, Informationen oder Darstellungen oder die kritische Interpretationsfähigkeit statistischer oder mathematischer Nachrichten (PIAAC Numeracy Expert Group 2009, S. 9).

\subsection{Zusammenhang von numeralen Kompetenzen und Weiterbildungsverhalten}

Aktuelle Forschungsbefunde zeigen diverse Zusammenhänge von Kompetenzniveaus und Weiterbildungsverhalten. Einerseits müssen Erwachsene mit geringen numeralen Kompetenzen mit Stigmatisierungen rechnen, was zu Zurückhaltung in der Teilnahme an Lernaktivitäten führen kann (Windisch 2016, S. 286). Nicht für Numeralität, aber für Literalität zeigt die LEO-Studie 2018, dass Befürchtungen, Anforderungen nicht gerecht zu werden, ein überdurchschnittlich häufig genannter Grund für die Nichtteilnahme an Weiterbildungen für Erwachsene mit geringen Kompetenzen sind (Dutz und Bilger 2020). Windisch (2016, S. 283-286) verweist auf unerkannte oder nicht als dringlich erachtete Lernbedarfe bei Erwachsenen mit geringer Numeralität. Dies weist auf den „Referenzgruppeneffekt“ (in der internationalen Forschung diskutiert als big-fish-little-pond-effect) hin, wonach die eigenen Fähigkeiten relativ zum Lernumfeld eingeschätzt werden (Trautwein und Lüdtke 2010). Andererseits belegen die Analysen auf Basis der britischen Birth Cohort Study (BCS70), dass Erwachsene mit numeralen Schwierigkeiten überdurchschnittliche Bestrebungen haben, ihre numeralen Kompetenzen zu verbessern (Carpentieri et al. 2009, S. $53 \mathrm{f}$.).

Darüber hinaus erklärt die enge Korrelation von Numeralität mit soziodemografischen Merkmalen, insbesondere mit niedriger formaler Bildung, einen Teil des Zusammenhangs von geringer Numeralität und geringem Weiterbildungsverhalten (ebd., S. 28f.). Dennoch lassen sich numerale Kompetenzen von Erwachsenen nur zum Teil durch Schulbildung erklären. Als gleichwertig erachtete Schulabschlüsse und Mathenoten gehen sowohl national als auch international mit unterschiedlichen numeralen Kompetenzniveaus einher; zudem zeigen sich auch im Erwachsenenalter numerale Kompetenzveränderungen durch formale, non-formale oder informelle Bildungsprozesse (Ausbildung, Hochschulstudium, Weiterbildung). Weiterhin geht geringe Numeralität häufig mit geringer Literalität einher; dennoch überschneiden sie sich nur zum Teil (Grotlüschen et al. 2016, S. 23). 
Ferner hängt Numeralität mit arbeitsmarktbezogenen Faktoren zusammen: Gering numeralisierte Erwachsene sind häufiger in niedrig qualifizierten Berufsfeldern beschäftigt und häufiger erwerbslos als Erwachsene mit hohen numeralen Kompetenzen (Carpentieri et al. 2009, S. 41 ff.; Rammstedt 2013, S. 134). Berufliche und auf den Erwerbsstatus bezogene Kontexte fördern und ermöglichen die Weiterbildungsteilnahme unterschiedlich stark (Wittpoth 2018, S. $1162 \mathrm{f}$.). Unter dem Begriff „low-skilled-trap“ wird die Abwärtsspirale von geringem (numeralen) Kompetenzniveau, erhöhtem Risiko von Arbeitslosigkeit oder Positionen im Niedriglohnbereich, geringeren Entwicklungsmöglichkeiten und der damit einhergehenden Gefahr des Kompetenzverlustes oder -stagnation beschrieben (Windisch 2016, S. 283). Dies wiederum wirkt sich auch auf die Art und Dauer der Weiterbildungsteilnahme aus.

\subsection{Geringe Numeralität und Weiterbildungsvolumen im deutschen Kontext}

Die Auswertungen der Kompetenzstudie PIAAC belegen den Matthäus-Effekt im Hinblick auf geringe Numeralität und geringe Weiterbildungsbeteiligung auch nach Kontrolle soziodemografischer Variablen (Grotlüschen et al. 2016, S. 96). Hinsichtlich der kompetenzbezogenen Verteilung des Weiterbildungsvolumens deutet sich dagegen ein entgegengesetzter Zusammenhang für Deutschland an, dieser wird jedoch nicht weiter diskutiert. Zumindest für Literalität bestätigt die LEO-Studie 2018, dass Erwachsene mit geringeren Kompetenzniveaus - trotz der unterdurchschnittlich häufigen Teilnahme - insgesamt mehr Zeit in Weiterbildungen verbringen als jene mit hohen Kompetenzen (Dutz und Bilger 2020).

Erklärungsansätze für die Effektumkehrung beim Weiterbildungsvolumen im deutschen Kontext bieten die Analysen zur berufsbezogenen Weiterbildung von Bläsche et al. (2017). Betriebliche, individuell berufsbezogene Weiterbildungen, Weiterbildungen der Bundesagentur für Arbeit oder die im Zuwanderungsgesetz geregelten Integrationskurse unterscheiden sich wesentlich hinsichtlich der Stundenintensität als auch der Adressatengruppen. Betriebliche Weiterbildungen machen zwar mit $70 \%$ den mit Abstand größten Weiterbildungsbereich aus, sie umfassen jedoch meist nur wenige Stunden (ebd., S. $12 \mathrm{f}$.).

Während die kürzeren betrieblichen Weiterbildungen und Bildungsurlaube häufiger von Hochqualifizierten und Führungskräften mit hohem Einkommen wahrgenommen werden (Anbuhl 2015), partizipieren gering Qualifizierte und Erwerbslose häufiger an den Maßnahmen der Bundesagentur für Arbeit. Letztere sind insbesondere stundenintensive Maßnahmen aus den Sozialgesetzbüchern II und III (Bläsche et al. 2017, S. 14). Zugewanderte partizipieren vor allem an den umfangreichen Integrationskursen (Grotlüschen und Haberzeth 2018, S. 553). Da geringe Numeralität überdurchschnittlich häufig mit Erwerbslosigkeit und geringem beruflichen Status einhergeht (Grotlüschen et al. 2016, S. 22-30), kann davon ausgegangen werden, dass die Befunde hinsichtlich des Weiterbildungsvolumens für Deutschland ähnlich ausfallen wie die Ergebnisse der LEO-Studie 2018 hinsichtlich der überdurchschnittlichen Weiterbildungszeit von Erwachsenen im unteren Kompetenzbereich. Die vermutete Umkehr des Matthäus-Effekts im Kontext von Numeralität wäre folglich mit stundenintensiven Weiterbildungsangeboten, die speziell gering qualifizierte Gruppen ansprechen, zu begründen. 


\subsection{Länderspezifische Weiterbildungsvolumina und Kompetenzen}

Weiterbildungen, die Numeralität gezielt fördern, sind in vielen Ländern Teil der Grundbildungspolitik (Knauber und Ioannidou 2016, S. 132). Knauber und Ioannidou (2016) stellen in ihren international vergleichenden Analysen zu Grundbildungspolitiken erhebliche Differenzen hinsichtlich des Verständnisses, der beteiligten Akteure und der inhaltlichen Ausgestaltung von Grundbildungspolitik zwischen den untersuchten Ländern (England, Österreich, Niederlande, Türkei) fest. Auch Umfang und Adressatengruppen von staatlich finanzierten Weiterbildungen sowie die Angebotsstrukturen für Personengruppen mit geringen Kompetenzen sind länderspezifisch sowie Ergebnis politischer Entscheidungen. Daher ist es nicht überraschend, dass international vergleichende Weiterbildungsstudien länderspezifische Differenzen hinsichtlich Weiterbildungsbeteiligung sowie in der kompetenzbezogenen Verteilung von Weiterbildungsstunden feststellen (Desjardins et al. 2006; Desjardins 2017, S. 185-187; Grotlüschen et al. 2016, S. 97-106; Markowitsch et al. 2013, S. 284). In Ländern mit hohen Partizipationsraten ist häufig ein hohes Weiterbildungsvolumen pro Teilnehmenden (Dänemark, Finnland, Niederlande, Norwegen) zu finden, andere Länder weisen niedrige Partizipationsraten bei gleichzeitig hohem Volumen der einzelnen Teilnehmenden auf (Irland, Slowenien) (Desjardins et al. 2006, S. 27-28). Hinsichtlich der formalen Bildung und der Weiterbildungsrate zeigen sich ähnliche Teilnahmemuster entsprechend des Matthäus-Effekts, jedoch variiert das Ausmaß der sozialen Selektivität von Weiterbildungssystemen enorm (Kaufmann et al. 2014, S. 39). Nähere Ausführungen mit Blick auf Numeralität fehlen allerdings bislang.

Die Ergebnisse der zahlreichen international vergleichenden Weiterbildungsstudien verweisen hinsichtlich des Umfangs und der Selektivität von Weiterbildungen auf Indikatoren auf der Makro- und Mesoebene (sogenannte Institutional Packages). Beispielsweise wirken sich ein hohes Bruttoinlandsprodukt pro Kopf (Markowitsch et al. 2013) und eine niedrige Arbeitslosenquote (Groenez et al. 2007) positiv auf das (betriebliche) Weiterbildungsvolumen aus. Die soziale Selektivität des Zugangs zu Weiterbildungen wird durch eine hohe Gewerkschaftsdichte (ebd.), hohe Arbeitsmarktförderung (Roosmaa und Saar 2012), hohe Bildungsausgaben (Dämmrich et al. 2014, S. 42; Martin und Rüber 2016, S. 167) sowie eine geringe soziale Ungleichheit (Lee und Desjardins 2019) verringert. Die häufig als sozialdemokratisch eingeordneten nordischen Länder sind in den genannten Punkten meist stärker aufgestellt (Esping-Andersen 1990). Desjardins weist auf die Rolle von Volkshochschulen und Erwachsenenbildungsverbänden für eine heterogene Weiterbildungsteilnehmerschaft hin, welche in nordischen Ländern überdurchschnittlich stark ausgebaut sind (Desjardins et al. 2006, S. 22f). Insgesamt variieren die Daten- und Bemessungsgrundlagen international vergleichender Weiterbildungsforschung (Kaufmann et al. 2014, S. 49), sie sind folglich nur begrenzt auf die hier untersuchten OECD-Länder übertragbar.

Aus dem Forschungstand lassen sich folgende Hypothesen ableiten (Tab. 1): 
Tab. 1 Hypothesen $1 \& 2$

Effektumkehr bei Weiterbildungsvolumina - in Stunden:

Hypothese 1 Erwachsene mit geringem numeralen Kompetenzniveau weisen ein höheres Weiterbildungsvolumen auf als Erwachsene mit hohen numeralen Kompetenzen

Effektumkehr im internationalen Vergleich:

Hypothese 2 Die Verteilung des Weiterbildungsvolumens von Erwachsenen entlang unterschiedlicher Kompetenzniveaus variiert zwischen den Ländern

\section{Quantitative Analyse}

\subsection{Datengrundlage \& Samplebeschreibung}

Auf Basis der PIAAC-Runden 1 und 2 (2011 bis 2016) können numerale Kompetenzen von Erwachsenen im erwerbsfähigen Alter (16 bis 65 Jahre) international verglichen werden. Zudem werden in PIAAC die Weiterbildungen differenziert nach der Dauer erfasst (Zabal et al. 2013). Die Stichprobengröße umfasst nach der Missing-Analyse insgesamt 122.682 Fälle und 23 Länder mit mindestens 4050 (Norwegen) und maximal 8563 (Polen) Beobachtungen (vgl. Tab. 2). Die Länder Australien, Estland, Kanada, Österreich, Russland, Zypern und die USA können nicht berücksichtigt werden, da sie mindestens eine der für die vorliegende Analyse relevanten Fragen nicht gestellt haben oder nur ein stark limitierter Zugang zu den Daten besteht.

\subsection{Operationalisierung}

Für die Untersuchung der Hypothesen wurde eine metrische abhängige Variable Weiterbildungsvolumen aus mehreren Fragen zur Weiterbildungsbeteiligung im nonformalen Bereich generiert. Es wurden die Kategorien „,betriebliche Schulungen/ Schulungen durch Vorgesetzte oder Kollegen“, „Fernunterricht/E-Learning“, „,Seminare/Workshops“, sowie „sonstige Kurse/Privatunterricht“ berücksichtigt. Hier werden nur Personen erfasst, die angaben, mindestens eine Stunde an einem der genannten non-formalen Bereiche in den 12 Monaten vor der Befragung teilgenommen zu haben.

Für die Messung von Numeralität wurde entsprechend der PIAAC-Konzeption eine kategoriale Variable mit vier Ausprägungen ( $1=$ Level 1 und geringer, $2=$ Level 2, 3=Level 3, 4=Level 4 und höher) auf Basis der in Zabal et al. (2013) beschriebenen Konzeption gebildet. In den Analysen wird Level 1 und geringer (geringe Numeralität) mit Level 4 und höher (hohe Numeralität) verglichen.

Soziodemografische und arbeitsbezogene Merkmale werden in Form von Kontrollvariablen in den Schätzungen berücksichtigt. Die Variable Bildungsabschluss basiert auf der ISCED-Klassifikation (1997) und wurde aufgrund geringer Fallzahlen und in Anlehnung an Rammstedt (2013, S. 98) im höchsten formalen Bildungsabschluss zu den Kategorien (1) niedriger Bildungsabschluss (ISCED 1,2 \& geringer), (2) mittlerer Bildungsabschluss (ISCED 3,4), (3) tertiärer Bildungsabschluss (ISCED 5,6) subsummiert. Die berufliche Tätigkeit basiert auf der ISCOSKIL4Variable, welche die Berufshauptgruppen der ISCO-Klassifikation (2008) in vier 
Tab. 2 Stichprobenverteilung

\begin{tabular}{|c|c|c|c|c|}
\hline Variable & $(n=122.682)$ & $\mathrm{SD}$ & $\begin{array}{l}\text { Numerales } \\
\text { Level } 1 \text { und } \\
\text { geringer } \\
n=27.986 \\
(25 \%)\end{array}$ & $\begin{array}{l}\text { Numerales } \\
\text { Level } 4 \text { und } \\
\text { darüber } \\
n=14.001 \\
(10,5 \%)\end{array}$ \\
\hline Weiterbildungsstunden & 153,31 & 2,7129 & 163,61 & 138,23 \\
\hline Weiterbildung $(1=$ ja $)$ & 0,39 & 0,0025 & 0,21 & 0,63 \\
\hline NumTestScore & 266,26 & 0,2903 & 185,48 & 346,87 \\
\hline \multicolumn{5}{|l|}{ Numerales Kompetenzlevel } \\
\hline Level $1 \&$ geringer & 25,00 & 0,2584 & & \\
\hline Level 2 & 33,52 & 0,3478 & & \\
\hline Level 3 & 31,03 & 0,2749 & & \\
\hline Level 4 \& 5 (Ref.-Kategorie) & 10,45 & 0,1569 & & \\
\hline Beschäftigt ( 1 = beschäftigt $)$ & 0,82 & 0,0012 & 0,53 & 0,84 \\
\hline Alter & 41,52 & 0,0249 & 43,96 & 38,98 \\
\hline Geschlecht (1 = männlich) & 0,50 & 0,0007 & 0,44 & 0,65 \\
\hline Migrationshintergrund $(1=\mathrm{Ja})$ & 0,14 & 0,0016 & 0,18 & 0,11 \\
\hline \multicolumn{5}{|l|}{ Formaler Bildungsabschluss } \\
\hline Niedrig (ISCED $1 / 2 \&$ geringer) & 0,27 & 0,0014 & 0,58 & 0,03 \\
\hline Mittel (ISCED 3/4) & 0,43 & 0,0018 & 0,33 & 0,32 \\
\hline Tertiär (ISCED 5/6) & 0,30 & 0,0013 & 0,09 & 0,66 \\
\hline \multicolumn{5}{|l|}{ Berufliche Tätigkeit } \\
\hline Seit 5 Jahren nicht tätig & 0,18 & 0,0016 & 0,31 & 0,07 \\
\hline Helfer- und Anlerntätigkeiten & 0,08 & 0,0013 & 0,14 & 0,02 \\
\hline $\begin{array}{l}\text { Fachlich ausgerichtete Tätigkei- } \\
\text { ten }\end{array}$ & 0,19 & 0,0020 & 0,22 & 0,10 \\
\hline Komplexe Spezialtätigkeiten & 0,26 & 0,0020 & 0,22 & 0,21 \\
\hline Hoch komplexe Tätigkeiten & 0,29 & 0,0019 & 0,10 & 0,60 \\
\hline \multicolumn{5}{|l|}{ Anzahl der Bücher } \\
\hline Max 25 Bücher & 0,42 & 0,0027 & 0,69 & 0,14 \\
\hline 26-100 Bücher & 0,31 & 0,0023 & 0,21 & 0,32 \\
\hline 101-200 Bücher & 0,13 & 0,0018 & 0,06 & 0,21 \\
\hline 201-500 Bücher, oder mehr & 0,15 & 0,0015 & 0,04 & 0,34 \\
\hline
\end{tabular}

Länder (n): Belgien (4597), Chile (4792), Dänemark (6725), Finnland (5057), Frankreich (6284), Deutschland (4838), Griechenland (4701), Großbritannien (8119), Irland (5716), Israel (4664), Italien (4352), Japan (4536), Korea (6194), Luxemburg (4821), Niederlande (4699), Norwegen (4050), Polen (8563), Slowakei (5214), Slowenien (4845), Spanien (5556), Schweden (4067), Tschechien (5319), Türkei 4973

Anforderungsstufen zusammenfasst: (1) Helfer- und Anlerntätigkeiten (2) fachlich ausgerichtete Tätigkeiten (3) komplexe Spezialtätigkeiten und (4) hoch komplexe Tätigkeiten (Führungs-, Manager- und akademische Tätigkeiten) (eigene Bezeichnung in Anlehnung an die Bundesagentur für Arbeit (2011, S. 22 ff.)). Die Variable Migrationshintergrund wurde gemäß dem statistischen Bundesamt aus dem Geburtsland und dem Immigrationsstatus der Eltern operationalisiert. Demnach wurden Erwachsene mit Migrationshintergrund erfasst, wenn ,,sie selbst oder mindestens 
eines ihrer Elternteile nicht mit deutscher Staatsbürgerschaft geboren sind“ (Statistisches Bundesamt 2018). Außerdem wurden die Anzahl der Bücher im Haushalt, das Geschlecht, sowie das Alter und der Beschäftigungsstatus ( 1 = beschäftigt, $0=$ unbeschäftigt/erwerbsunfähig) in die Analyse einbezogen.

\subsection{Modellspezifikationen}

Um den prognostizierten positiven Zusammenhang von Weiterbildungsvolumen mit Numeralität zu überprüfen, wurde sich einfacher und multipler linearer Regressionsmodelle (Ordinary Least Squares, OLS) bedient, da ein linearer Zusammenhang zwischen den unabhängigen Variablen und der abhängigen Variable unterstellt wird und die abhängige Variable Weiterbildungsvolumen metrisch skaliert ist. Die Analysen wurden mit STATA und dem Replication Estimation Modul (Repest) von Francesco Avvisati und François Keslair durchgeführt. Das Repest-Modul erlaubt die Berechnung korrekter Schätzer und Standardfehler unter Berücksichtigung der plausiblen Werte (plausible Values) und des komplexen Stichprobendesigns.

\section{Ergebnisse}

\subsection{Deskriptive Ergebnisse}

Es geben $39 \%$ (gerundete Werte) der Erwachsenen aus der Stichprobe an $(n=52.711)$, an einer oder mehreren non-formalen Weiterbildungen teilgenommen zu haben und zwar im Durchschnitt $153 \mathrm{~h}$ im Jahr. Bei Betrachtung der numeralen Kompetenzen fällt auf, dass $11 \%$ der Erwachsenen der Stichprobe ein numerales Kompetenzlevel 4 oder 5 aufweisen, $25 \%$ erreichen lediglich ein numerales Kompetenzlevel 1 oder geringer. Im Durchschnitt erreichen die Erwachsenen der untersuchten Länder 266 Punkte (Kompetenzstufe 2).

Im Hinblick auf die Weiterbildungsbeteiligung ist es auf Basis des eindeutigen Forschungsstandes wenig überraschend, dass sich die Beteiligung an non-formaler Weiterbildung zwischen der obersten und der untersten Kompetenzgruppe wesentlich unterscheidet: Während die Beteiligungsquote bei Erwachsenen mit geringer Numeralität lediglich $21 \%$ beträgt, liegt sie bei Erwachsenen mit dem höchsten Numeralitätsniveau mit $63 \%$ um mehr als das Dreifache höher. Ein entgegengesetztes Bild zeigt sich bei der Weiterbildungsdauer der Teilnehmenden. Erwachsene mit geringer Numeralität (164h) liegen deutlich über dem Länder-Durchschnitt (153h) und dem der Erwachsenen mit Kompetenzlevel 4 und darüber (138h).

\subsection{Regressionsergebnisse}

Da davon auszugehen ist, dass diese Verteilung je nach nationalen Rahmenbedingungen variiert, wird die Weiterbildungsdauer im Folgenden differenziert nach Kompetenzniveau pro Land dargestellt.

Die in Abb. 1 dargestellten linearen Regressionskoeffizienten sind als durchschnittliche Differenzen im Weiterbildungsvolumen von Erwachsenen mit geringer 


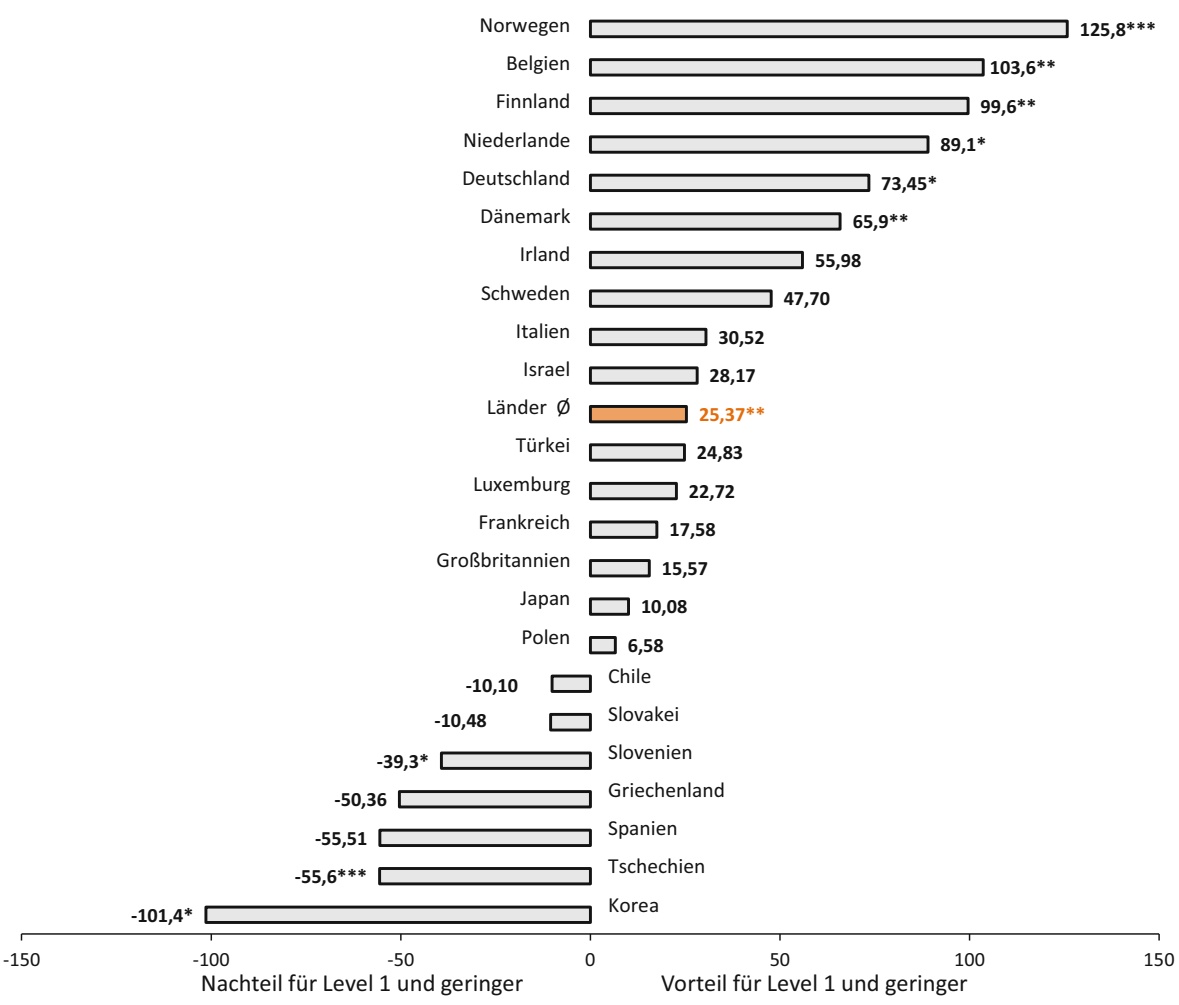

Abb. 1 Differenzen im Weiterbildungsvolumen (in Stunden) zwischen Erwachsenen mit numeralen Kompetenzen im Level 1 und geringer und Erwachsenen mit numeralen Kompetenzen im Level 4 und darüber. OLS-Modell, ohne Kontrollvariable

Numeralität gegenüber Erwachsenen mit hoher Numeralität (Referenzgruppe) je Land zu interpretieren. In den OLS-Modellen stellt das Weiterbildungsvolumen (in Stunden) die abhängige Variable dar, Kontrollvariablen wurden nicht aufgenommen. Liegen die Balken im positiven Wertebereich (rechts), weisen Erwachsene mit geringer Numeralität durchschnittlich mehr Weiterbildungsstunden auf als Erwachsene mit hoher Numeralität.

Die Befunde zeigen für Deutschland, dass Erwachsene mit geringer Numeralität im Durchschnitt 73,5 Stunden mehr Zeit in Weiterbildungen verbringen als Erwachsene mit hoher Numeralität. Hinsichtlich der Verteilung von Weiterbildungszeit zu Gunsten der Gruppe mit geringen numeralen Kompetenzen steht Deutschland damit auf dem fünften Platz. Auch der Länder-Durchschnitt fällt signifikant positiv aus: Erwachsene mit hoher Numeralität nehmen durchschnittlich 25,4 Stunden mehr Weiterbildungszeit als die Vergleichsgruppe.

Hinsichtlich des Ländervergleichs zeigt sich der vermutete positive Zusammenhang zwischen geringer Numeralität und Weiterbildungsvolumen in 16 der 23 untersuchten Länder. Signifikant ist dieser Zusammenhang in sechs Ländern - neben Deutschland auch in Belgien und den Niederlanden (zwei der drei Benelux-Länder) sowie Finnland, Norwegen und Dänemark (drei von vier nordeuropäischen Län- 


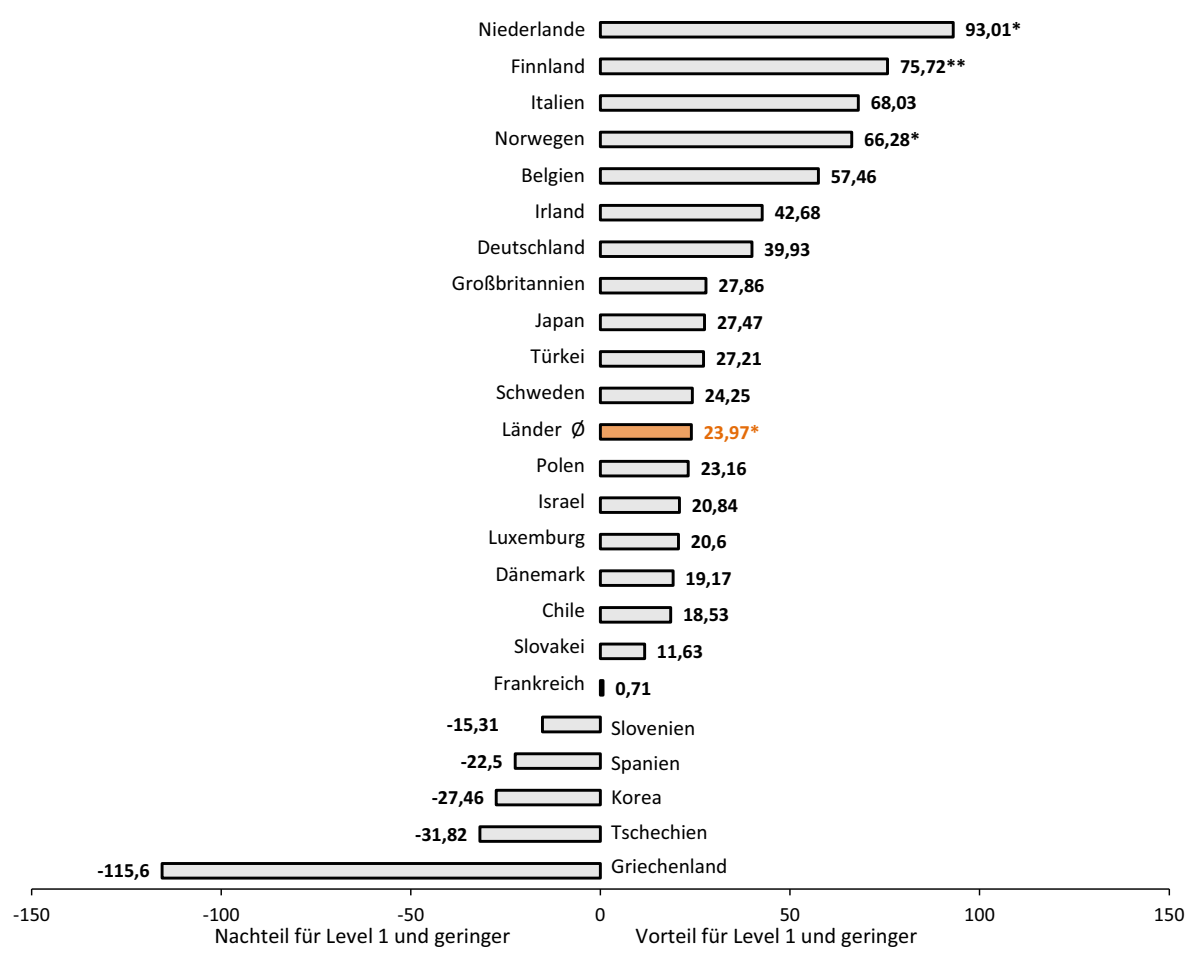

Abb. 2 Differenzen im Weiterbildungsvolumen (in Stunden) zwischen Erwachsenen mit numeralen Kompetenzen im Level 1 und geringer und Erwachsenen mit numeralen Kompetenzen im Level 4 und darüber. OLS-Modell, mit Kontrollvariable

dern). Dagegen sind Erwachsene mit geringer Numeralität in drei von vier Ländern der EU-Osterweiterung (Tschechien, Slowenien, Slowakei) sowie in zwei von vier südeuropäischen Ländern (Griechenland, Spanien) hinsichtlich des Weiterbildungsvolumens benachteiligt.

In Abb. 2 werden die Koeffizienten der multiplen OLS-Regressionsmodelle für Erwachsene mit geringer Numeralität unter Berücksichtigung der soziodemografischen Kontrollvariablen (Tab. 2) dargestellt.

Für Deutschland zeigt sich bei Berücksichtigung der Kontrollvariablen, dass Erwachsene mit geringer Numeralität fast 40 Stunden mehr Zeit in Weiterbildungen verbringen als jene mit hoher Numeralität. Die Stundendifferenz liegt damit in Deutschland immer noch über dem Länderdurchschnitt, verringert sich jedoch um fast die Hälfte zum Modell ohne Kontrollvariablen und wird insignifikant. Im OECD-Durchschnitt bleibt der signifikant positive Zusammenhang zwischen geringer Numeralität und dem Weiterbildungsvolumen bestehen. Mit Blick auf den internationalen Vergleich zeigt sich unter Berücksichtigung der Kontrollvariablen über alle Länder hinweg eine Verminderung des positiven Zusammenhangs zwischen geringer Numeralität und Weiterbildungsvolumen. Die Signifikanzen bleiben in drei Ländern bestehen: Niederlande, Finnland und Norwegen. Interessanterweise verändern sich die Effekte in den Ländern, die deutlich über dem Länder-Durchschnitt 
liegen, tendenziell zu Gunsten der Erwachsenen mit hohen Kompetenzen. In den Ländern, in denen geringere Numeralität negativ mit dem Weiterbildungsvolumen zusammenhängt, verändert sich dagegen der Einfluss leicht zum Vorteil für Erwachsene mit geringer Numeralität (mit Ausnahme von Griechenland). In Chile und der Slowakei dreht sich der Effekt um, bleibt aber geringfügig. Überraschend sind die Ergebnisse für Dänemark: Unter Berücksichtigung soziodemografischer Merkmale verringert sich der signifikante Vorteil von Erwachsenen mit geringer Numeralität im Weiterbildungsvolumen um mehr als zwei Drittel (von 65,9 auf 19,2 Stunden) gegenüber jenen mit hoher Numeralität. Dänemark liegt damit als einziges nordeuropäisches Land unterhalb des Länderdurchschnitts.

Insgesamt zeigt der Ländervergleich mit Kontrollvariablen ein ähnliches Muster wie die Befunde ohne Kontrollvariablen: In den nordeuropäischen Ländern, den Benelux-Ländern und Deutschland besuchen Erwachsene mit geringer Numeralität tendenziell länger Weiterbildungen als jene mit hoher Numeralität. Der positive Zusammenhang von geringer Numeralität und hoher Weiterbildungszeit ist jedoch lediglich in drei Ländern signifikant. In den süd- und osteuropäischen Ländern sowie in Korea und Chile ist dieser Zusammenhang geringer oder entgegengesetzt.

Die schrittweise Untersuchung des Zusammenhangs zunächst ohne und anschließend mit Kontrollvariablen verdeutlicht Folgendes: Soziodemografische und arbeitsbezogene Variablen erklären erstens einen Teil des Zusammenhangs von geringen numeralen Kompetenzen und Weiterbildungsvolumen. Zweitens hat geringe Numeralität in den Niederlanden, Norwegen, Finnland sowie im OECD-Durchschnitt einen eigenständigen positiven Effekt auf das Weiterbildungsvolumen. Drittens sind Weiterbildungsstunden in der überwiegenden Mehrheit der untersuchten OECD-Länder zu Gunsten der Teilnehmenden mit geringer Numeralität verteilt, jedoch sind die Unterschiede zwischen gering und hoch numeralisierten Erwachsenen meist nicht signifikant.

\section{Diskussion der Ergebnisse}

Die vorliegenden Ergebnisse sind im Hinblick auf den aktuellen Forschungsstand differenziert zu betrachten. Der Matthäus-Effekt lässt sich für das Weiterbildungsvolumen im Kontext von Numeralität bei der großen Mehrheit der Länder nicht belegen. Viel mehr deutet sich eine Effektumkehr an: Die Erwachsenen mit geringen numerale Kompetenzen, eben jene, für die Förderung und Kompetenzentwicklung am ehesten wichtig erscheinen, verbringen im Betrachtungszeitraum von einem Jahr mehr Stunden in Weiterbildungen als jene mit hohen Kompetenzen. Signifikant sichtbar wird dieser Zusammenhang insbesondere für die nordischen Länder Norwegen, Finnland sowie für das Benelux-Land Niederlande - auch unter Berücksichtigung sozialdemografischer und berufsbezogener Faktoren. Für diese Länder kann Hypothese 1 bestätigt werden. Allerdings belegen die vorliegenden Analysen auch den von Grotlüschen et al. (2016) gefundenen kumulativen Bildungseffekt hinsichtlich der Weiterbildungsquote. D.h. Erwachsene mit den höchsten numeralen Bildungsbedarfen nehmen insgesamt verhältnismäßig selten an Weiterbildungen 
teil. Die Effektumkehr trifft folglich lediglich für gering numeralisierte Erwachsene $\mathrm{zu}$, die tatsächlich an Weiterbildungen teilnehmen.

In der Summe deuten die ländervergleichenden Ergebnisse systematische Differenzen hinsichtlich der Volumina-Verteilung an. Hypothese 2 kann damit bestätigt werden. Folgende Tendenzen konnten aufgezeigt werden: In den nordeuropäischen und Benelux-Ländern ist das Weiterbildungsvolumen eher zu Gunsten der Erwachsenen mit geringer Numeralität verteilt (in drei Ländern signifikant), sie liegen in der Verteilung über dem OECD-Durchschnitt. Dies knüpft an die Befunde von Desjardins et al. (2006) an: Die nordischen Länder weisen hohe Partizipationsraten bei gleichzeitig hoher Stundenzahl pro Teilnehmenden auf. Korea sowie die süd- und osteuropäischen Länder liegen unter dem OECD-Durchschnitt und weisen Verteilungen der Weiterbildungsvolumina zum Nachteil von Erwachsenen mit geringer Numeralität oder lediglich geringfügige, nicht signifikante Vorteile auf.

Der gefundene Zusammenhang von Numeralität und Weiterbildungsverhalten ist teilweise auf die enge Korrelation von numeralen Kompetenzen mit arbeitsbezogenen und soziodemografischen Merkmalen zurückzuführen. Erwachsene mit unterschiedlichen Kompetenzniveaus haben Zugang zu unterschiedlichen Weiterbildungsangeboten.

In Deutschland werden die kürzeren betrieblichen Weiterbildungen und Bildungsurlaube eher von Erwachsenen wahrgenommen, deren berufliche Tätigkeiten ohnehin schon hohe numerale Kompetenzanforderungen stellen und einen hohen Gebrauch an numeralen Praktiken verlangen (Bläsche et al. 2017, S. 10f.). Dies sind insbesondere Tätigkeiten im Führungs-, Management- und Wissenschaftsbereich (Rammstedt 2013, S. 145 f.). Dagegen greifen für Erwachsene mit geringer Numeralität eher die langfristigen Weiterbildungsangebote der Arbeitsmarkt- und Integrationspolitik oder berufsbildende Weiterbildungen wie Aufstiegsfortbildungen.

Der für Finnland, Norwegen und die Niederlande aufgezeigte eigenständige $\mathrm{Nu}$ meralitätseffekt kann nachfrageseitig so ausgelegt werden, dass Erwachsene mit geringen Kompetenzen durchaus eine hohe Lernmotivation haben (vgl. Carpentieri et al. 2009, S. 53 f.). Hinsichtlich der Angebotsseite kann interpretiert werden, dass die staatliche Arbeits- und Integrationsförderung ihre Zielgruppen mit langfristigen Angeboten durchaus erreicht, allerdings lediglich innerhalb der weiterbildungsaktiven Population. Hohe Volumina betreffen überwiegend Erwachsene, deren numeraler Kompetenzentwicklungsbedarf am höchsten ist.

Die gefundenen internationalen Differenzen entlang geografischer Regionen knüpfen an die Befunde internationaler Weiterbildungsforschung an. Sie verweisen auf die Rolle wohlfahrtsstaatlicher Politik und den Wohlstand eines Landes. Konkret lässt sich feststellen, dass die Länder, die in den Analysen durchgängig Spitzenreiter in der Verteilung des Weiterbildungsvolumens zu Gunsten derjenigen mit dem höchsten Bedarf sind (Niederlande, Finnland, Norwegen), auch höhere öffentliche Bildungsausgaben und ein höheres Bruttoinlandsprodukt pro Kopf aufweisen als der OECD-Durchschnitt. Die Länder, in denen der Matthäus-Effekt tendenziell auch für das Weiterbildungsvolumen zutrifft (Slowenien, Tschechien, Spanien, Griechenland, Korea) haben dagegen ein unterdurchschnittliches Niveau an öffentlichen Bildungsausgaben und Wohlstand (OECD factbook 2013, 2014). Ähnliche Muster deuten sich für die staatliche Arbeitsmarktförderung und die Höhe 
öffentlicher Sozialausgaben an. Die länderbezogenen Verteilungen von Weiterbildungsvolumen verweisen auf die von Knauber und Ioannidou (2016) diskutierten Unterschiede in der Ausgestaltung von Grundbildungspolitik (Grundbildungsverständnis, Zuständigkeiten und Handlungsspielräume). Diese sind maßgebend für das Ziel, den Umfang und die Adressatengruppe staatlicher Weiterbildungsmaßnahmen und stellen Bestandteile wohlfahrtsstaatlicher Systeme dar.

\section{Desiderata}

Die bisher vorgelegte Analyse verweist auf geografische Regionen und lässt erste Schlüsse über die Rolle wohlfahrtsstaatlicher Systeme und damit verbundener Grundbildungspolitiken im Kontext von Numeralität und Weiterbildungsvolumen zu. Zur vertieften Aufschlüsselung der Ergebnisse müssten nunmehr die Makro- und Mesoebene insbesondere der Arbeitsmarkt- und Integrationspolitik in die Analysen einfließen - etwa mittels Mehr-Ebenen-Modellen und Strukturgleichungsanalysen.

Eine Verknüpfung von vergleichender Bildungsforschung und vergleichender Politikforschung sowie deren systematische Anwendung auf die aktuelle Ausgestaltung politischer Ökonomien von OECD-Ländern kann als ausstehender Forschungsschritt gesehen werden. Die Überblicksarbeiten von Kaufmann et al. (2014) und Knauber (2017) bieten dabei gute Anknüpfungspunkte.

\section{Limitationen der Ergebnisse}

Die Ergebnisse basieren auf PIAAC-Daten (2011 bis 2016), weshalb jüngste sozialstaatliche Veränderungen, wie etwa Migrationsbewegungen seit 2015 und Reformen in den Weiterbildungs- und Arbeitsförderungsgesetzen, nicht abgebildet werden können. Auf Basis dieser Querschnittsanalyse kann nicht von kausalen Zusammenhängen gesprochen werden, da Zeitdiskontinuitäts- und Endogenitätsprobleme nicht vollständig auszuschließen sind. Teils geringe Fallzahlen im Kompetenzlevel 4 und darüber und damit einhergehende vergrößerte Standardfehler können in einigen Ländern (z. B. in Spanien) zu einer Unterschätzung des Zusammenhangs zwischen Numeralität und Weiterbildungsvolumen geführt haben.

Als relevant eingestufte Kontrollvariablen, wie Einkommen und Weiterbildungsfinanzierung, wurden nicht in die Analyse aufgenommen. In PIAAC liegt nur das individuelle Einkommen, nicht aber das Haushaltseinkommen vor. Zudem weist PIAAC zwar die Unterstützung durch Arbeitgeber, nicht aber die staatliche Finanzierung aus.

Weiterhin liegen in PIAAC keine Informationen darüber vor, ob an einer Weiterbildung teilgenommen wurde, um numerale Praktiken auszubauen oder gezielt eine Weiterbildung gewählt wurde, in der diese Fähigkeiten nicht gebraucht werden. Für die weitere Forschung wäre ein Bezug zu den Inhalten der Weiterbildungen sinnvoll. 


\section{Implikationen für Politik und Praxis}

Die Bedeutung von Numeralität ist abhängig von sozialen Kontexten, sie reicht von kritischer Rezeption von Statistiken bis zum Umgang mit der Altersvorsorge. Für gewerbliche, technische, kaufmännische Berufsfelder aber auch für hochqualifizierte Tätigkeiten im Führungs-, Management- und Wissenschaftsbereich sind numerale Kompetenzen entscheidend. Nicht zuletzt beeinflusst Numeralität aufgrund der Verwobenheit mit sozialen Kontextfaktoren und genuinen Kompetenzeffekten das Bildungsverhalten von Erwachsenen.

Politik und Praxis erleben seit Jahrzehnten eine wiederkehrende, scheinbar erfolglose Suche nach der Überwindung des Matthäus-Effekts. Beispielsweise fragte Hans Tietgens schon 1964, warum nur wenige Industriearbeiter in die Volkshochschulen kommen (Tietgens 1978). Politische und praktische Versuche besserer Ansprache, so der Anschein, bleiben wirkungslos. Das ist schon hinsichtlich der Teilnahmequoten nicht durchgehend der Fall - Ältere und Frauen holen beispielsweise in spezifischen Segmenten auf (Bilger et al. 2017) - aber es trifft speziell und immer wieder die in Bezug auf Grundbildung weniger kompetenten Gruppen. Daraus leitet sich die Frage ab, ob bildungspolitische Programmlinien mit entsprechenden Praxisprojekten überhaupt Erfolgschancen haben.

Bei Betrachtung der Weiterbildungsstunden zeigt sich nunmehr zumindest in einigen Ländern eine Effektumkehr. Ausgebaute Arbeitsmarktförderung und öffentliche (Weiter-)Bildungsausgaben zeigen sich länderübergreifend als relevante politische Größen für die (Um-)Verteilung von Weiterbildungszeit (Dämmrich et al. 2014; Martin und Rüber 2016; Roosmaa und Saar 2012).

Gerade längerfristige Bildungsangebote spielen zudem bei der Entwicklung eines bildungsnahen Habitus eine große Rolle. Es ist insofern denkbar, dass eine langfristige Weiterbildungsteilnahme zu wiederkehrender Teilnahme führt. Dies wurde schon hinsichtlich des relativ kurzen Bildungsurlaubs als „Initialzündung“ (Zeuner 2017, S. 14) diskutiert und in Teilen auch nachgewiesen (ebd.).

Allerdings sind langfristige staatliche Programme teilweise verpflichtend und zielen nicht genuin auf Bildung, sondern auf Beschäftigung und Integration ab. Entsprechende Kritik wird immer wieder geäußert (Bonna 2018; Heinemann 2018).

Open Access Dieser Artikel wird unter der Creative Commons Namensnennung 4.0 International Lizenz veröffentlicht, welche die Nutzung, Vervielfältigung, Bearbeitung, Verbreitung und Wiedergabe in jeglichem Medium und Format erlaubt, sofern Sie den/die ursprünglichen Autor(en) und die Quelle ordnungsgemäß nennen, einen Link zur Creative Commons Lizenz beifügen und angeben, ob Änderungen vorgenommen wurden.

Die in diesem Artikel enthaltenen Bilder und sonstiges Drittmaterial unterliegen ebenfalls der genannten Creative Commons Lizenz, sofern sich aus der Abbildungslegende nichts anderes ergibt. Sofern das betreffende Material nicht unter der genannten Creative Commons Lizenz steht und die betreffende Handlung nicht nach gesetzlichen Vorschriften erlaubt ist, ist für die oben aufgeführten Weiterverwendungen des Materials die Einwilligung des jeweiligen Rechteinhabers einzuholen.

Weitere Details zur Lizenz entnehmen Sie bitte der Lizenzinformation auf http://creativecommons.org/ licenses/by/4.0/deed.de. 


\section{Anhang}

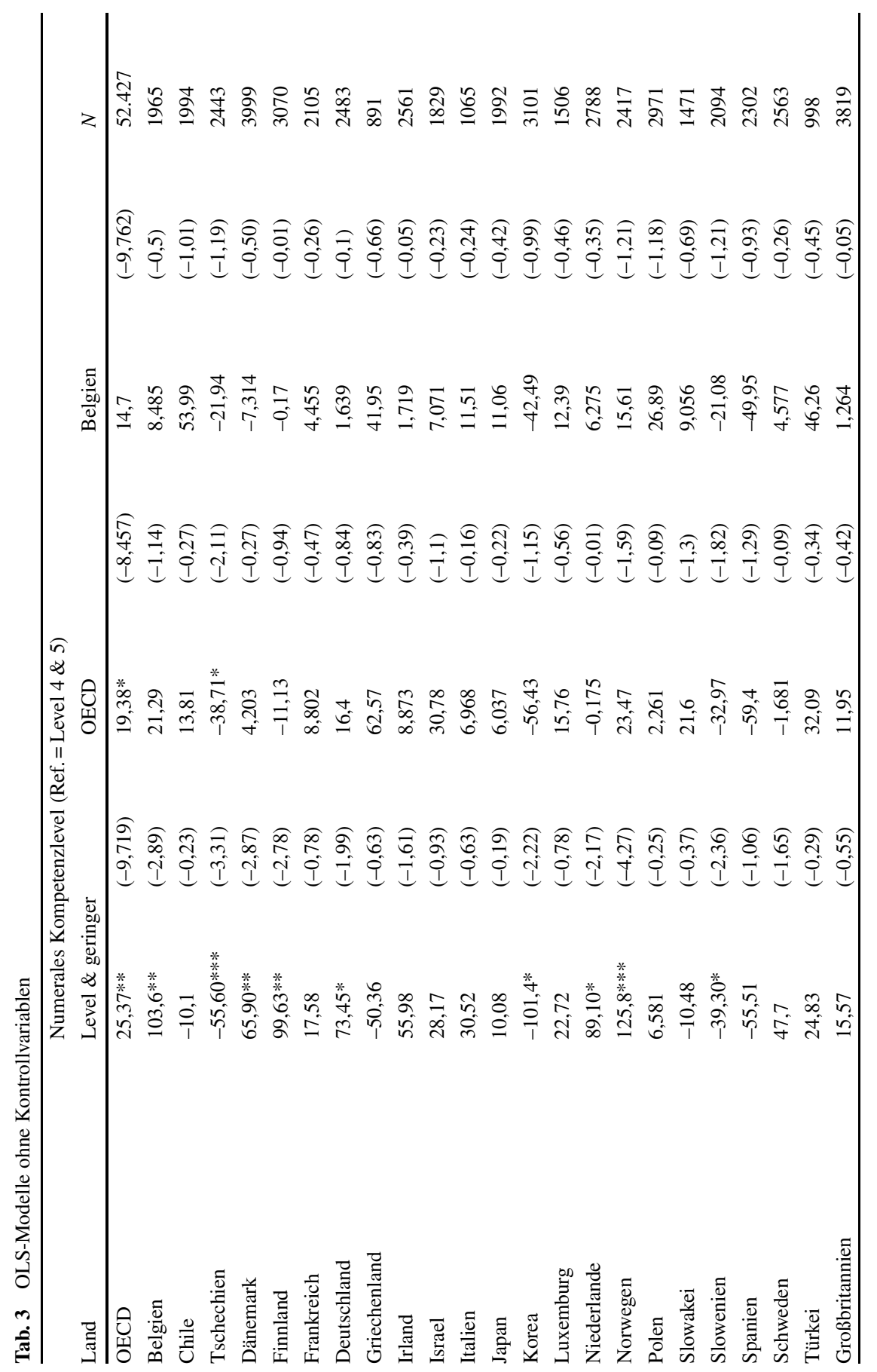




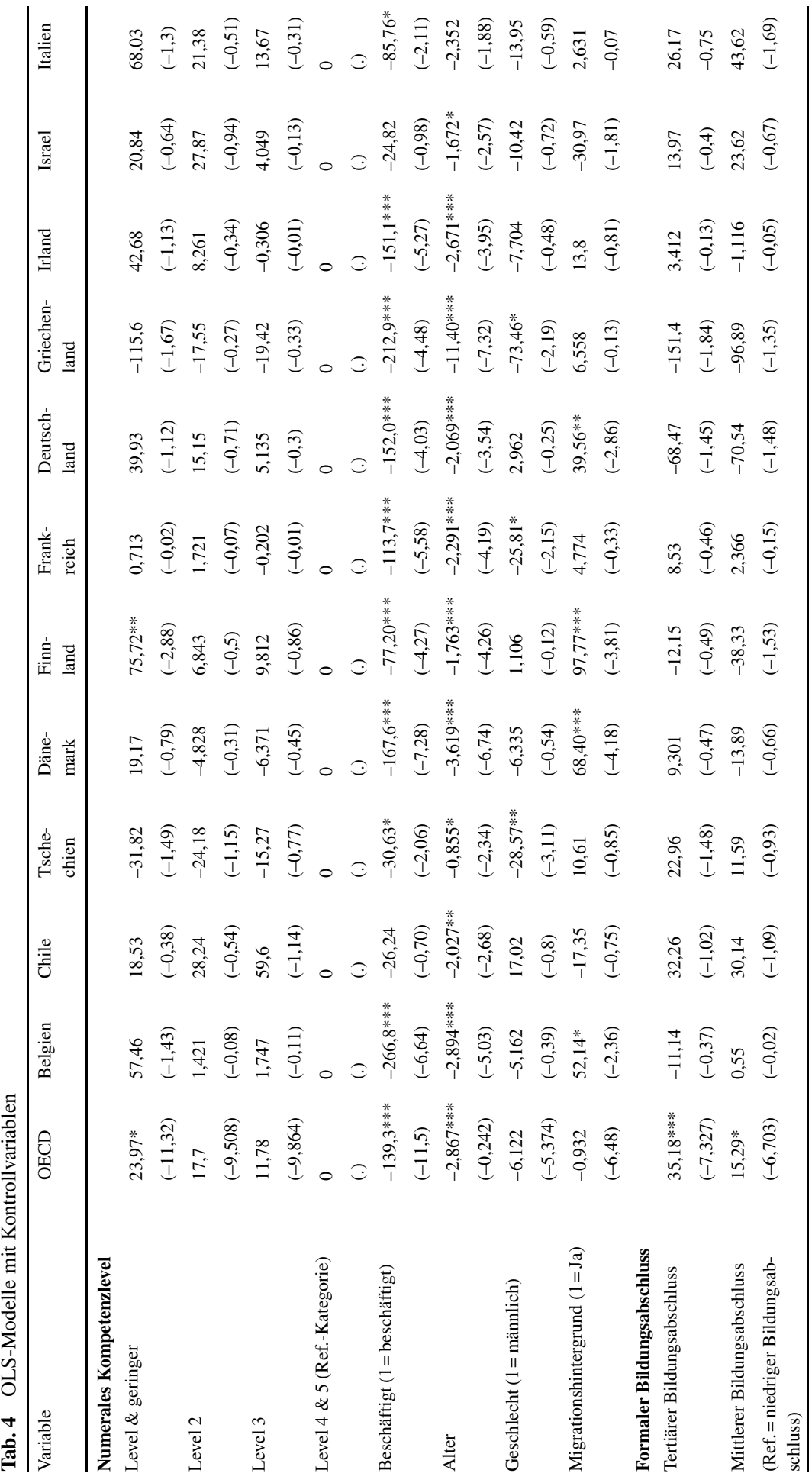




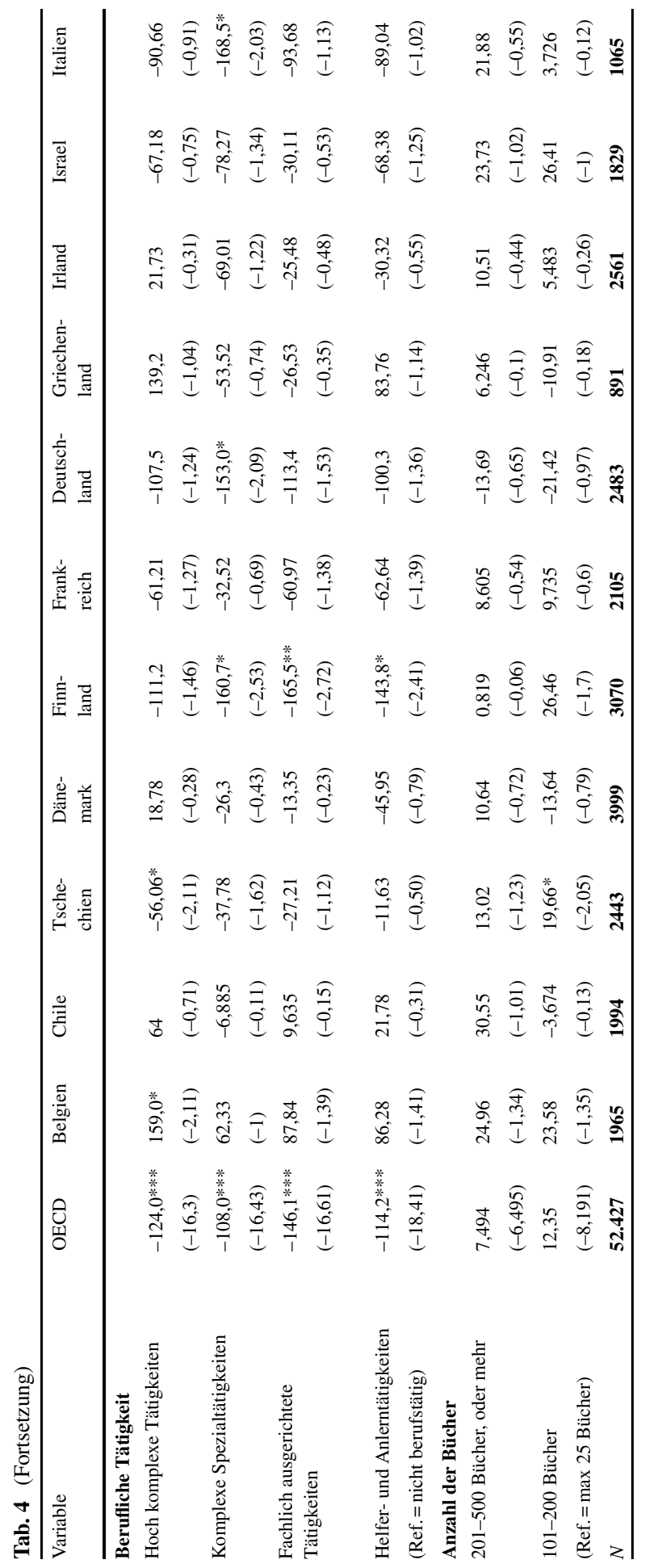




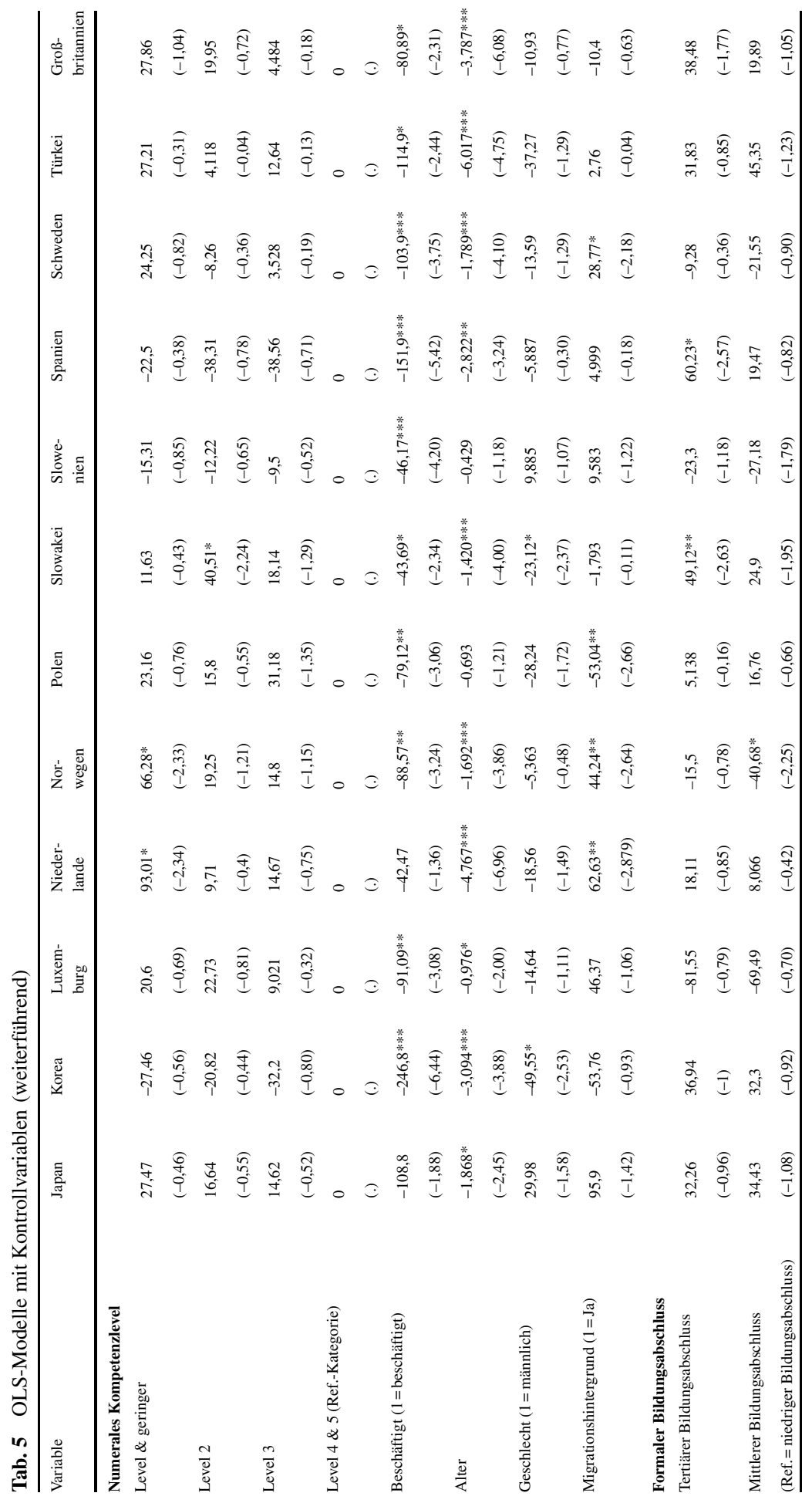




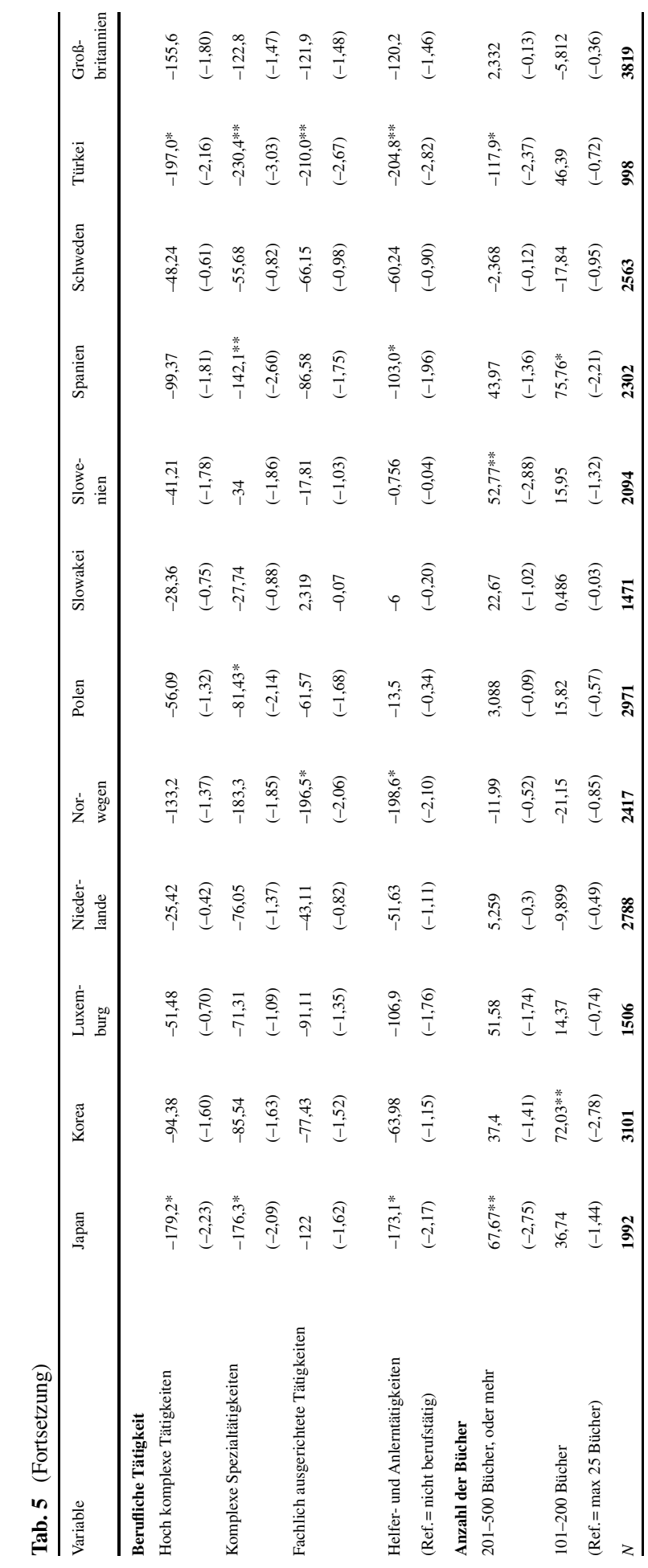




\section{Literatur}

Anbuhl, M. (2015). Die Zwei-Klassen-Gesellschaft. DGB-Analyse zur sozialen Spaltung in der Weiterbildung. Berlin: DGB-Bundesvorstand.

Banks, J., O’Dea, C., \& Oldfield, Z. (2010). Cognitive function, numeracy and retirement saving trajectories. The Economic journal, 120(548), 381-410.

Bilger, F., Behringer, F., Kuper, H., \& Schrader, J. (Hrsg.). (2017). Weiterbildungsverhalten in Deutschland 2016. Ergebnisse des Adult Education Survey (AES) (DIE Survey - Daten und Berichte zur Weiterbildung). Bielefeld: wbv. https://doi.org/10.3278/85/0016w

Bläsche, A., Brandherm, R., Eckhardt, C., Käpplinger, B., Knuth, M., Kruppe, T., Kuhnhenne, M., \& Schütt, P. (2017). Qualitätsoffensive strukturierte Weiterbildung in Deutschland. Forschungsförderung Working Paper Nr. 25. Düsseldorf: Hans Böckler Stiftung.

Bonna, F. (2018). Berufliche Zukunftsvorstellungen Langzeitarbeitsloser. Eine biographieanalytische Untersuchung. Erwachsenenbildung und lebensbegleitendes Lernen - Forschung \& Praxis, V. 31. Bielefeld: wbv media.

Bundesagentur für Arbeit (2011). Systematischer und alphabetischer Teil mit Erläuterungen. Klassifikation der Berufe 2010, Bd. 1.

Carpentieri, J. D., Lister, J., \& Frumkin, L. (2009). Adult numeracy. A review of research. London: Institute of Education University of London.

Dämmrich, J., Vono de Vilhena, D., \& Reichart, E. (2014). Partipicipation in adult learning in Europe: The impact of country-level and individual characteristics. In H.-P. Blossfeld, E. Kilpi-Jakonen, D. Vono de, Vilhena \& S. Buchholz (Hrsg.), Adult Learning in Modern Societies. Cheltenham: Edward Elgar Publishing.

Desjardins, R. (2017). Political economy of adult learning systems. Comparative study of strategies, policies and constraints. London: Bloomsbury Academic.

Desjardins, R., Rubenson, K., \& Milana, M. (2006). Unequal chances to participate in adult learning: international perspectives. Paris: UNESCO: International Institute for Educational Planning.

Disney, R., \& Gathergood, J. (2013). Financial literacy and consumer credit portfolios. Journal of Banking \& Finance, 37(7), 2246-2254. https://doi.org/10.1016/j.jbankfin.2013.01.013.

Dutz, G., \& Bilger, F. (2020). Bildungshintergrund und Weiterbildungsverhalten gering literalisierter Erwachsener. In A. Grotlüschen \& K. Buddeberg (Hrsg.), LEO 2018 - Leben mit geringer Literalität. Bielefeld: wbv media.

Eckert, T. (2010). Methoden und Ergebnisse der quantitativ orientierten Erwachsenenbildungsforschung. In R. Tippelt \& A. v. Hippel (Hrsg.), Handbuch Erwachsenenbildung/Weiterbildung (S. 263-279). Wiesbaden: VS.

Esping-Andersen, G. (1990). The three worlds of capitalism. New Jersey: Princton University Press.

French, D., \& McKillop, D. (2015). Financial literacy and over-indebtedness in low-income households. International Review of Financial Analysis, 48, 1-11. https://doi.org/10.1016/j.irfa.2016.08.004

Groenez, S., Desmedt, E., \& Nicaise, I. (2007). Participation in lifelong learning in the EU-15: the role of macro-level determinants. Gent: Paper for the ECER Conference.

Grotlüschen, A., \& Haberzeth, E. (2018). Weiterbildungsrecht. In R. Tippelt \& A. v. Hippel (Hrsg.), Handbuch Erwachsenenbildung/Weiterbildung. Springer Reference Sozialwissenschaften. (S. 543-563). Wiesbaden: Springer VS.

Grotlüschen, A., Mallows, D., Reder, S., \& Sabatini, J. (2016). Adults with low adults with low proficiency in literacy or numeracy. OECD Education Working Papers. Paris: OECD.

Heinemann, A. M. B. (2018). Alles unter Kontrolle? Zeitschrift für Weiterbildungsforschung, 41(1), 79-92. https://doi.org/10.1007/s40955-018-0106-8.

Kaufmann, K., Reichart, E., \& Schömann, K. (2014). Der Beitrag von Wohlfahrtsstaatsregimen und Varianten kapitalistischer Wirtschaftssysteme zur. Erklärung von Weiterbildungsteilnahmestrukturen bei Ländervergleichen. In P. Gonon (Hrsg.), International-vergleichende Forschung zur Erwachsenenbildung. Report. Zeitschrift für Weiterbildungsforschung, 37. Jahrgang, Heft 2/2014. (S. 39-54). Bielefeld: W. Bertelsmann.

Knauber, C. (2017). International-vergleichende Forschung zur Bildungspolitik: Literatur-Review theoretischer Ansätze und Perspektiven der vergleichenden Politikwissenschaft. Zeitschrift für Weiterbildungsforschung, 40(2), 171-198.

Knauber, C., \& Ioannidou, A. (2016). Politiken der Grundbildung im internationalen Vergleich - Von der Politikformulierung zur Implementierung. Zeitschrift für Weiterbildungsforschung- Report, 39(2), 131-148. https://doi.org/10.1007/s40955-016-0071-z. 
Lee, J., \& Desjardins, R. (2019). Inequality in adult learning and education participation: the effects of social origins and social inequality. International Journal of Lifelong Education, 38(3), 339-359. https://doi.org/10.1080/02601370.2019.1618402.

Lusardi, A., \& de Bassa Scheresberg, C. (2013). Financial Literacy and High-Cost Borrowing in the United States. Cambridge: National Bureau of Economic Research.

Markowitsch, J., Käpplinger, B., \& Hefler, G. (2013). Firm-provided training in Europe and the limits of national skills strategies. European Journal of Education, 48(2), 281-291. https://doi.org/10.1111/ ejed.12030.

Martin, A., \& Rüber, I. E. (2016). Die Weiterbildungsbeteiligung von Geringqualifizierten im internationalen Vergleich - Eine Mehrebenenanalyse. Zeitschrift für Weiterbildungsforschung, 39(2), 149-169. https://doi.org/10.1007/s40955-016-0060-2.

OECD (2013). factbook. Economic, environmental and social statistics. Paris: OECD.

OECD (2014). factbook. Economic, environmental and social statistics. Paris: OECD.

PIAAC Numeracy Expert Group (2009). PIAAC numeracy: a conceptual framework. OECD Education Working Papers. Paris: OECD.

Rammstedt, B. (Hrsg.). (2013). Grundlegende Kompetenzen Erwachsener im internationalen Vergleich. Ergebnisse von PIAAC 2012. Münster: Waxmann.

Rau, A. (2016). Nichtformale und informelle Lerngelegenheiten im Zusammenspiel mit familiären Sozialisationsmerkmalen und leistungsrelevanten Einstellungen. Hamburg: Universität Hamburg.

Reder, S. (2005). Literacy and the life-course. reflect. The Magazine Of NRDC, (ISSUE 2), 16-17.

Roosmaa, E.-L., \& Saar, E. (2012). Participation in non-formal learning in EU-15 and EU-8 countries: demand and supply side factors. International Journal of Lifelong Education, 31(4), 477-501. https:// doi.org/10.1080/02601370.2012.689376.

Schmidt-Lauff, S. (2018). Zeittheoretische Implikationen in der Erwachsenenbildung. In R. Tippelt \& A. v. Hippel (Hrsg.), Handbuch Erwachsenenbildung/Weiterbildung (S. 319-338). Wiesbaden: Springer.

Statistisches Bundesamt (2018). Bevölkerung und Erwerbstätigkeit. Bevölkerung mit Migrationshintergrund-Ergebnisse des Mikrozensus 2017. Fachreihe 1, Bd. 2.2. Wiesbaden: Statistisches Bundesamt.

Tietgens, H. (1978). Warum kommen wenig Industrie-Arbeiter in die Volkshochschule? (1964). In W. Schulenberg (Hrsg.), Erwachsenenbildung. (S. 98-174). Darmstadt: Wissenschaftliche Buchgesellschaft.

Trautwein, U., \& Lüdtke, O. (2010). Referenzgruppeneffekte. In W. Bos (Hrsg.), Schulische Lerngelegenheiten und Kompetenzentwicklung. Festschrift für Jürgen Baumert (S. 11-30). Münster: Waxmann.

UNESCO (2016). Education 2030. Incheon declaration and framework for action for the implementation of sustainable development goal 4.

Windisch, H.C. (2016). How to motivate adults with low literacy and numeracy skills to engage and persist in learning: a literature review of policy interventions. International Review of Education, 62(3), 279-297. https://doi.org/10.1007/s11159-016-9553-x.

Wittpoth, J. (2018). Beteiligungsregulation in der Weiterbildung. In R. Tippelt \& A. v. Hippel (Hrsg.), Handbuch Erwachsenenbildung/Weiterbildung (S. 1149-1172). Wiesbaden: Springer.

Yasukawa, K. (2018). The workplace as a site for learning critial numeracy practice. In K. Yasukawa, A. Rogers, K. Jackson \& B. Street (Hrsg.), Numeracy as Social Practise. Global and Local Perspectives (S. 225-240). New York: Routledge.

Zabal, A., Martin, S., Klaukien, A., Rammstedt, B., Baumert, J., \& Klieme, E. (2013). Grundlegende Kompetenzen der erwachsenen Bevölkerung in Deutschland im internationalen Vergleich. In B. Rammstedt (Hrsg.), Grundlegende Kompetenzen Erwachsener im internationalen Vergleich. Ergebnisse von PIAAC 2012 (S. 31-76). Münster: Waxmann.

Zeuner, C. (2017). Bildungszeit für alle? Hamburg: Helmut Schmidt Universität.

Publisher's Note Springer Nature remains neutral with regard to jurisdictional claims in published maps and institutional affiliations. 